\title{
Joint-Action Coordination of Redundant Force Contributions in a Virtual Lifting Task
}

\section{Jurjen Bosga and Ruud G. J. Meulenbroek}

\begin{abstract}
In this study we investigated redundancy control in joint action. Ten participantpairs (dyads) performed a virtual lifting task in which isometric forces needed to be generated with two or four hands. The participants were not allowed to communicate but received continuous visual feedback of their performance. When the task had to be performed with four hands, participants were confronted with a redundant situation and between-hand force synergies could, in principle, be formed. Performance timing, success rates, cross-correlations, and relative phase analyses of the force-time functions were scrutinized to analyze such task-dependent synergies. The results show that even though the dyads performed the task slower and less synchronized in the joint than in the solo conditions, the success rates in these conditions were identical. Moreover, correlation and relative phase analyses demonstrated that, as expected, the dyads formed between-participant synergies that were indicative of force sharing in redundant task conditions.
\end{abstract}

Key Words: coordination, redundancy, coupling, human, joint action

One of the key challenges of cognitive neuroscience is to understand the principles that govern redundancy control, i.e., how biological systems extract from a large set of available control dimensions the minimum number of dimensions that is needed to act adequately. The issue was first addressed by Bernstein (1967) who in studies of multi-joint task performance identified "the degrees of freedom problem." Since then, a variety of mechanisms have been demonstrated by means of which people "solve" redundant control problems. For example, the coupling of actuators into motor synergies is one strategy to simplify coordination problems (Cole \& Abbs, 1986; Santello, Flanders \& Soechting, 1998; D'Avella, Saltiel \& Bizzi, 2003; Ivanenko, Grasso, Zago, Molinari, Scivoletto, Castellano, Macellari \& Lacquaniti, 2003). For single actuator movements it has been suggested that the central nervous system uses motion plans that are defined in terms of joint angles (Uno, Kawato \& Suzuki, 1989; Nakano, Imamizu, Osu, Uno, Gomi, Yoshioka \& Kawato, 1999; Rosenbaum, Loukopoulos, Meulenbroek, Vaughan \& Engelbrecht, 
1995. Rosenbaum, Meulenbroek, Vaughan \& Jansen, 2001) or hand position coordinates (Morasso, 1981; Flash \& Hogan, 1985; Viviani \& Flash; 1995). Combined with, respectively, the minimum torque change and minimum jerk principles, the neuromotor system is supposed to solve the redundancy problem quasi-automatically. Regardless of the nature of the reference systems in which the preparation of hand displacements takes place at various levels of the neuromotor system, experiments have shown that movements are always planned in such a way that the execution of these plans is robust against the variability that is inherent to the motor system (Harris, 1998; Harris \& Wolpert, 1998).

Because lifting an object together requires the cooperation of two people, it is an attractive task to study coordination in joint task performance. First, it allows us to determine the extent to which joint-action coordination is reactive or proactive. In addition, it allows us to investigate how groups deal with redundancy. How multiple degrees of freedom are contained in joint-action situations has hardly been studied before and we reasoned that a controlled study of a virtual joint-lifting task could fill this gap. Before going into the details of the experimental task we designed for this purpose, we first will summarize what is known about redundancy control in complex task performance by individuals.

\section{Intrapersonal Coordination}

During static and dynamic force production tasks, individual finger forces show signs of mutual dependence. For example, when a person is asked to exert force with a single digit, force occurs at other digits as well (the enslaving effect; for a review, see Schieber \& Santello, 2004). Also, when a person exerts maximal force with two fingers simultaneously, the total force produced by the two fingers is smaller than the sum of the maximal force production of both fingers separately (force deficit effects; see Li, Latash \& Zatsiorsky, 1998; Danion, Schoner, Latash, Li, Scholz \& Zatsiorsky, 2003). These force deficit effects of finger coordination resemble the well-known phenomenon of bilateral deficit (Koh, Grabiner \& Clough, 1993; Oda \& Moritani, 1994, 1995; Hakkinen, Kraemer \& Newton, 1997). Findings in splitbrain patients (Gazzaniga \& Sperry, 1966), cortical lesions (Wyke, 1971) and of reduction of EMG after TMS of the motor cortex (Ferbert, Priori, Rothwell, Day, Colebatch \& Marsden, 1992) suggest that, for simultaneous movements and dualtask performance by the individual, bilateral interhemispheric inhibition mediated through interhemispheric fibers plays some role in reducing motor performance, as indicated by measurements such as movement initiation, force, and speed (Ohtsuki, 1994). Furthermore, systematic covariations between finger forces indicate that the coupling of fingers into motor synergies is an important control strategy for isometric force production of the digits of one hand. For example, Santello and Soechting (2000) investigated the control of full-hand grasping by measuring finger contact forces when participants lifted, held, and replaced a manipulandum. They showed that there are two bosic temporal synergies in the control of isometric forces by the digits of the hand: one in which the forces of all of the digits vary to comparable degrees in the same direction, i.e., in phase, and a second, in which the forces exerted by some of the fingers are $180^{\circ}$ out of phase with the forces exerted by other fingers. The first synergy suggests the presence of a "common drive" to all of the extrinsic finger muscles and is presumed to suit coarse control of forces, whereas the second one suggests a finer "force stabilizing" control strategy (see also Li et al., 1998). In the present study, we were concerned with characterizing the coordination of finger forces that are in operation when two people perform a force production task together.

\section{Interpersonal Coordination}

Needless to mention is, as the proverb says "Many hands make light work," that there are situations conceivable in which joint action is compulsory for obvious reasons, e.g. when the to-be-lifted object is too heavy or its dimensions are too awkward to be handled alone. Otherwise, group members acting together are, in general, at a disadvantage when compared to actions carried out by an individual. In particular, it has been shown that performance is seriously hampered in joint action if the task at hand requires individuals to time their actions contingent upon those of others (Burstedt, Edin \& Johansson, 1997; Schmidt, Bienvenu, Fitzpatrick \& Amazeen, 1998; Knoblich \& Jordan, 2003).

Burstedt et al. (1997) investigated the coordination of fingertip forces when two participants (i.e., a dyad) lifted one object as compared to when this task was carried out unimanually or bi-manually by a single participant. Even though grasp stability was accomplished in a similar manner, synchronizing the actions of the fingertips of two participants remained poor in comparison to performance by a single participant, even after practice. Also force generation, prior to lifting the object, was executed slower by two participants $(723 \mathrm{~ms})$ than bimanual (459 $\mathrm{ms}$ ) and unimanual (456 ms) performance by one participant. Likewise, replacing the object after the "replace" command was given, was carried out more slowly in trials involving two participants, notwithstanding the observation that the trial was always performed successfully.

Both joint and single action can, in principle, rely on the same control mechanisms. Research conducted on rhythmic tasks has shown that the same dynamical fundamentals that govern the coordination of the movements of the limbs of individuals also govern the coordination of rhythmic movements of dyads who share visual information of their performance (Schmidt, Carello \& Turvey, 1990; Schmidt \& Turvey, 1994; Amazeen, Schmidt \& Turvey, 1995). Even though the preferred interpersonal coordination regimes were weaker than intra-personal between-limb coordination (Schmidt et al., 1998), these studies nevertheless showed that group members clearly tend to synchronize their actions when they can see each other's movements.

A different approach to model synchronized action coordination between individuals is to propose that participants (actors) plan and execute their actions in relation to what they anticipate the partner will do. Knoblich and Jordan (2003) have shown that groups indeed possess the ability to use and learn an anticipatory coordination strategy based on visual information even when they encounter more coordination problems than individuals due to increasing task demands. However, groups were able to enhance their performance if they were provided an external cue regarding the state of the partner's action alternative. Although the availability of such information did not affect performance initially, group performance became more similar to individual performance in later trials. 


\section{Experimental Paradigm and Predictions}

We developed an isometric force virtual lifting task with real-time feedback that allowed us to assess joint-action coordination under redundant task conditions. Our virtual lifting task (see Figure 1, left panel) was performed by participants individually (solos) or in pairs (dyads) who were asked to generate isometric forces. The task consisted of generating an upward pressure with the left and right index finger on load cell transducers (lifting phase) after which these forces needed to be stabilized and maintained for a 2-s period (holding phase). The aim of our study was to contrast control mechanisms of intra-personal and inter-personal coordination and focus on essential features of force sharing in a lifting task.

Fast haptic feedback loops from tactile afferents of the fingertips $(\sim 65 \mathrm{~ms}$; Johansson \& Birznieks, 2004) with which individuals or dyads applied pressure on both sensors allowed them to monitor force modulations of their own contribution to the task. Individuals could also rely on relative slow online visual feedback ( 135 ms; Carlton, 1981; Saunders \& Knill, 2003) of the bar displacements on the computer screen to perceive the consequences of their actions. Dyads, however, only received relatively slow online visual feedback of their partners' actions, no haptic feedback (see Rosenbaum et al., 2006 for reverse conditions). We therefore expected between-limb intra-personal coordination to be stronger than inter-personal coordination regimes resulting in enhanced performance in controlling the tilt of the bar. Even though we expected proactive coordination in joint action to be weaker than in single action, as has been demonstrated by Schmidt et al. (1998), our prediction implies that the coupling of effectors into task specific units would be informationally based.

In performing the isometric lifting task with four hands, i.e. when the system was overspecified, dyads received fast haptic feedback of their own contribution to the task and only relative slow online visual feedback of the consequences of the actions they jointly brought about. In redundant conditions, however, the output to the lifting task was composed of both actors' actions thereby masking the individual contribution to the task. This means that actions carried out by one partner were not directly observable for the other. A viable way for the actors to anticipate and execute their actions based on visual feedback of the composite movements of their joint action is for the partners to adapt to the dynamics of these composed (superimposed) movements. The way that partners can learn about the dynamics of the joint contribution to the task is by forming an internal model of the joint dynamics of the action of which they are part of (cf. Atkeson, 1989; Jordan, 1994). Conditt, Gandolfo and Mussa-Ivaldi (1997) have demonstrated the presence of adaptive processes in the control of multijoint arm movements in point-to-point reaching movements in a velocity-dependent force field. They suggested that adaptation to a novel force field occurs by creating an "internal model" of this field. We expected actors to adapt to the dynamics of their joint contribution as expressed by increasing stable interactions of balancing the bar and we therefore took systematic covariations between the redundant (supernumerary) force-producing hands in redundant joint-action conditions to reflect between-participant synergies that are brought about by adaptive processes in sharing the task.

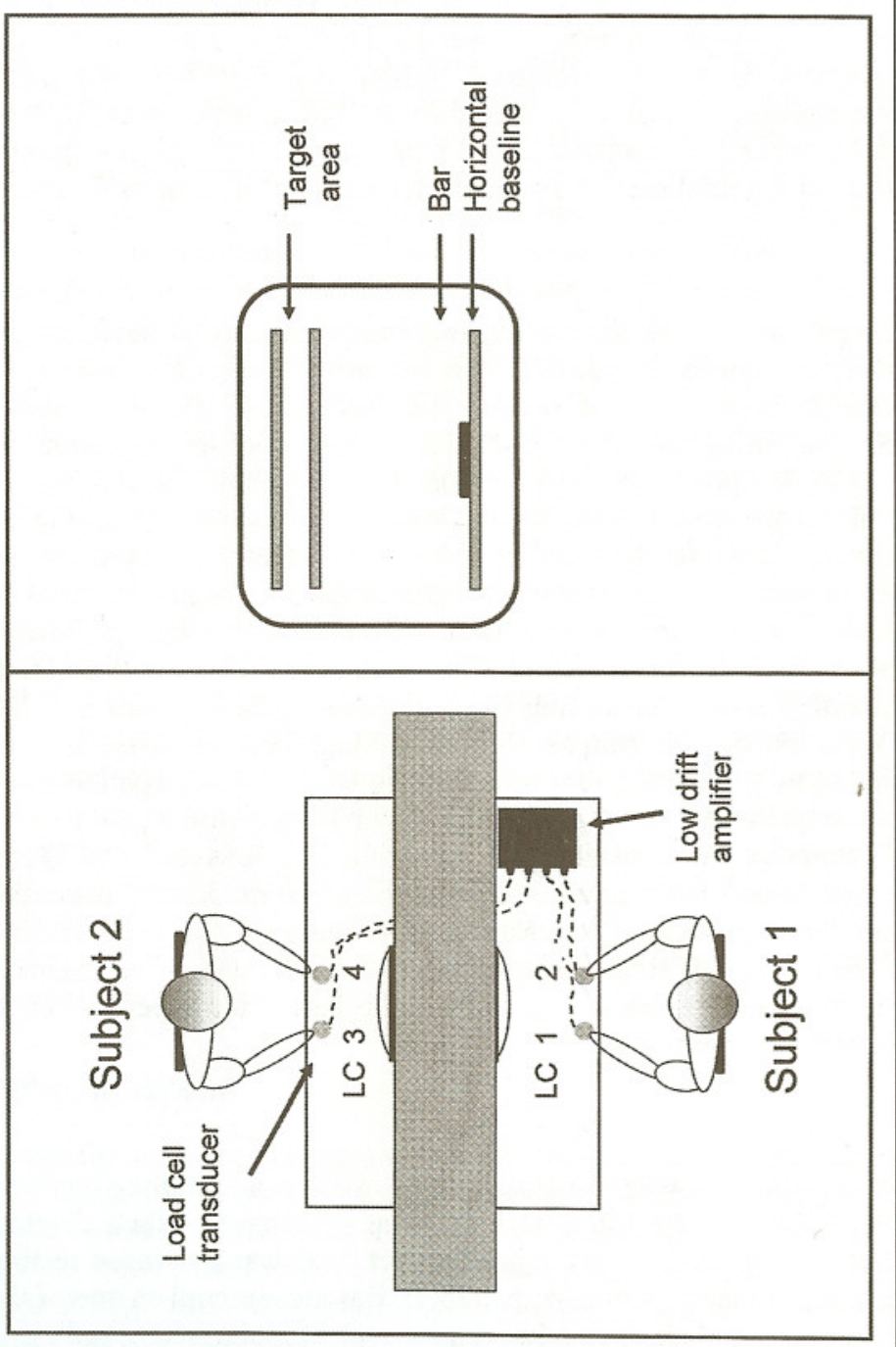




\section{Method}

\section{Participants}

Twenty right-handed students ( 4 male and 16 female) from the University of Nijmegen participated. Handedness was determined using a modified version of the Edinburgh Handedness Inventory (Oldfield, 1971). Their mean age in years and months was 22.4 (in years; months - range: 15-11; SD: 3.3). All participants had normal hearing and normal or corrected-to-normal vision and none had motor problems. All participants gave their informed consent. They were rewarded for their participation with either course credits or payment of 12 Euros. Experimental procedures followed the APA guidelines for the ethical treatment of human participants.

\section{Apparatus}

To perform the experimental task, the participants were randomly paired and were seated comfortably on adjustable chairs at opposite ends of the setup, vis-à-vis, at freestanding tables that were separated by a solid free-standing screen (see Figure 1, left panel). The virtual lifting task was displayed on two 17" CRT monitors (refresh rate: $75 \mathrm{~Hz} ; 1,024$ by 768 pixels) that were placed at eye level and at a comfortable distance on the table squarely in front of each participant. Two load cell transducers were mounted upside-down on the edge of each table and interspaced at $20 \mathrm{~cm}$. Force applied to the load cell resulted in changes of the electrical resistance of strain gauges housed in the load cell transducer (type BC302, DS Europe, Milan, Italy). The output from the load cell was amplified with a low-drift instrumentation amplifier and sampled in epochs starting from stimulus onset and ending 6,000 $\mathrm{ms}$ later at $1,500 \mathrm{~Hz}$. Force data from sensors 1 and 3 and force data from sensors 2 and 4 were linked by calculating the sum of the force output. The paired force data sets were resampled (at $75 \mathrm{~Hz}$ ), smoothed, and amplified with a gain of 375 . The resampled data served as displacement data for the left (sensors 1 and 3 ) and right (sensors 2 and 4 ) end of the bar and were recorded. The duration of the entire loop of the resampled data from one measurement to the next was $13.33 \mathrm{~ms}$ and the time lag between force input and drawing the bar on the display was on average $2.70 \mathrm{~ms}$. A zero force measurement just before the start of the experiment was obtained to calibrate the zero point.

\section{Task}

The participants were not allowed to communicate in any way but they received real-time visual feedback of the lifting task that was presented on their display. On these displays a black colored rectangular object (bar) was displayed resting on a horizontal baseline (see Figure 1, right panel). Bar movements on one of the displays were mirror imaged, thereby mimicking a real-life joint lifting task.

Participants could control the amount of lift (vertical position) and the tilt (rotation) of the bar in the frontal plane by exerting an upward pressure with their left and right index finger on load cell transducers. By distributing the applied force evenly over the load cell transducers they could ensure that the bar remained horizontally oriented. The amount of force required to lift the bar into the target area was similar whether the task was executed with one, two, or four hands. By keeping the target force constant across all conditions, we complied with the "Many hands make light work" principle.

\section{Procedure}

Before the experiment started, each participant was first allowed to become familiar with exerting force on the load cell transducers and the resulting movements of the bar. The setup allowed the participants to produce an isometric force with their index fingers on the sensors while their hands rested comfortably in their laps. Following this brief practice period they received written and verbal instructions.

Participants completed 11 blocks of 10 repetition trials, twice, a total of 220 trials per experiment. Each trial block consisted of a possible combination of action (single or joint), hands (two or four) and dexterity [left $(\mathrm{L})$ or right $(\mathrm{R})]$. This implied that the actors performed the action either alone with one hand ( $\mathrm{L}$ or R) or two hands (LR) or jointly with either two (LL, LR, RL, and RR) or four hands, amounting to $4+2+4+1=11$ blocks. The 11 blocks were presented randomly. In the task conditions in which a lifting force was applied to only one end of the bar (one-handed single actions and two-handed joint actions with mixed dexterity), only the vertical position of the bar needed to be controlled. In these conditions the rotation of the bar was fixed (to horizontal). Before each trial block, both participants were presented with graphic images of left/right hands on their displays to inform the participants with which combination of hands the lifting task was to be performed. Each trial started with a constant foreperiod of $1,500 \mathrm{~ms}$ in which the color of the bar changed from red to orange and finally to black (the go-signal) that indicated the start of a 6-s recording period. As soon as the visual go-signal was presented the participants were expected to move the bar towards a target position (Figure 1, right panel) and retain the position of the bar between the target's upper and lower boundaries for a 2-s period. The bar's color changed to green if the performance was successful; otherwise it remained black. At each trial completion, the final position of the bar remained visible for $3 \mathrm{~s}$ to allow for knowledge of results.

\section{Data Analysis}

Before the analysis, nine trials were excluded due to corrupted data. Furthermore, as we focused our study on comparable task constraints between individuals and dyads, we excluded the unimanual individual condition from further analysis because it did not require the actors to balance the bar. The force-time data $(1,500 \mathrm{~Hz})$ and resampled position-time data $(75 \mathrm{~Hz})$ were filtered with a second-order Butterworth, zero phase lag, low-pass filter with a cut-off frequency of $12 \mathrm{~Hz}$. For each trial, the filtered trajectory of the bar displacements (in pixels) and bar-orientation (in degrees) were derived. The data analyses focused on overall performance success rate, on variables that were visible to both actors in task space, and variables that were only perceivable to each actor individually defined in force space. 


\section{Performance}

Success rate was the percentage of trials that the participants completed successfully, i.e., if the bar was held in the target area for $2,000 \mathrm{~ms}$.

\section{Task Space}

Four phases were identified for each trial: latency, lift, stabilization, and hold phase (see also Figure 2). The latency phase was the time delay between the visual go-signal $($ for $\mathrm{t}=0$ ) and the averaged time indices of the onset of sampled force production of the hands involved in the relevant task condition and was determined by means of a computer search procedure (see the Appendix). The lift phase was defined as the time between the end of the latency phase and the first time at which both bar-ends were displaced above the lower limit boundary of the target (234 pixels). The stabilization phase was defined as the time between the lift phase and the time that the bar was contained for $2,000 \mathrm{~ms}$ in the target area (hold phase). The stabilization phase following the lifting of the bar was identified to exclude instabilities following the lift.

The difference in absolute time (ms) of the movement onset between the left and right end of the bar was calculated (to capture the degree of synchronization in movement initiation) and the variability of bar orientation as amount of rotation (in degrees) that the bar deviated from the horizontal orientation $\left(0^{\circ}\right)$ was calculated from the discrepancy between the resampled position-time data of the left and right end of the bar. Negative values indicated a deviation in a clockwise direction and positive values indicated a deviation in a counterclockwise direction. Bar orientation was a measure of unbalanced force generation acting on the left and right bar-end. Because the force output was directly coupled to the position on the computer screen, one Newton of force difference scaled as $\sim 5.74^{\circ}$ tilt angle. Cross-correlation functions of the bar-end displacement functions during the lift, stabilization, and hold phases were used to establish the relationship between the left and right end of the bar displacements. Time-lags were detected by an automatic search procedure for the local minima and maxima of the cross-correlation function of positional changes between the left and right side of the bar during the subsequent trial phases. We calculated the time between the extremes (minima or maxima) that occurred within each trial phase and calculated the mean cycle time (ms) per phase. We considered that the cycle time characterized the repetitive tuning of positional changes of the bar-ends within each trial phase. The means $(\mathrm{M} \phi)$ and standard deviations (STD $\phi$ ) of the continuous relative phase signals of the positionchange function were calculated by using Batschelet's (1981) procedure involving circular statistics (see Meulenbroek et al., 1998) for the lift and hold phases to determine the phase difference and the stability of the phase relationships of the resulting positional changes of the bar-ends. The 10 repetitions were collapsed into three grouped repetitions (GR) for which GR1 consisted of repetition 1 to 3; GR2 contained repetitions 4 to 6 , and the remaining four repetitions ( 7 to 10 ) were allocated to GR3. Furthermore, the bar-orientation function was subsequently subjected to time-series an lysis during the lift and hold phases by means of autocorrelation functions to reveal any systematic fluctuations in time. Time-lags were detected by a semi-automatic search procedure for relevant first local minima or the first zero crossing if the first local minimum was unavailable. The time lags were doubled to estimate cycle durations and converted to Hz. The temporal features of these autocorrelation functions were taken to reflect the dynamics of corrective movements.

\section{Force Space}

In force space, kinetic parameters were derived from the load cells to capture the degree of coordination between forces generated by individuals performing with two hands and by dyads performing the task with two and all four hands. The amount of force that was applied to each load cell was recorded and converted to Newtons. Between-participant force productions were further determined for redundant and non-redundant force relationships (see caption of Figure 1, left panel). Between-participant redundant force productions described relationships between supernumerary force productions whereby two hands jointly resulted in a displacement of either the left or right end of the bar. By contrast, betweenparticipant non-redundant force production described relationships between force productions that were not supernumerary whereby two hands resulted in a combined left/right or right/left displacement of the bar and thus acted complementary by nature of the task constraints. Cross-correlations during the lift, stabilization, and hold phases were used to establish the within-participant and between-participant (redundant and non-redundant joint actions) force-time relationship between force productions of the hands.

\section{Statistical Evaluation}

Sign tests were used to evaluate the degree to which observed differences between successful and unsuccessful performance was statistically significant across solos and dyads. We used paired samples $t$ tests to evaluate the differences between the 1 -actor/2-hands and 2-actors/2-hands conditions. We also used paired samples $t$ tests in the 2-actor/4-hands condition to statistically evaluate the cross-correlations and the mean and standard deviations of the relative phase functions of positional changes between grouped repetitions during the lift and hold phases. The critical value for Pearson's $r$ was set at the .05 level. Furthermore, for statistical evaluation of the correlation functions, the Pearson's $r$ values were transformed to the normally distributed variable $z$ by means of Fisher's $z$ transform. Bonferroni corrections were applied whenever multiple tests were conducted.

\section{Results}

Figure 2 shows plots of the force-time functions and their resultants (top panel) and the position-time functions of the left and right bar-end (bottom panel) observed in a trial that was performed successfully. These data are typical for the task execution of the individual participants as well as the dyads. The top panel shows the data that were extracted from a condition in which two actors performed the trial with both hands. Actor 2 led the way in force initialization $(\sim 150 \mathrm{~ms})$ and Actor 1 followed $(\sim 290 \mathrm{~ms})$. After positioning the bar into the target area, both actors maintained forces at approximately the same level with both hands while forces between supernumerary contributions changed reciprocally. This process occurred 


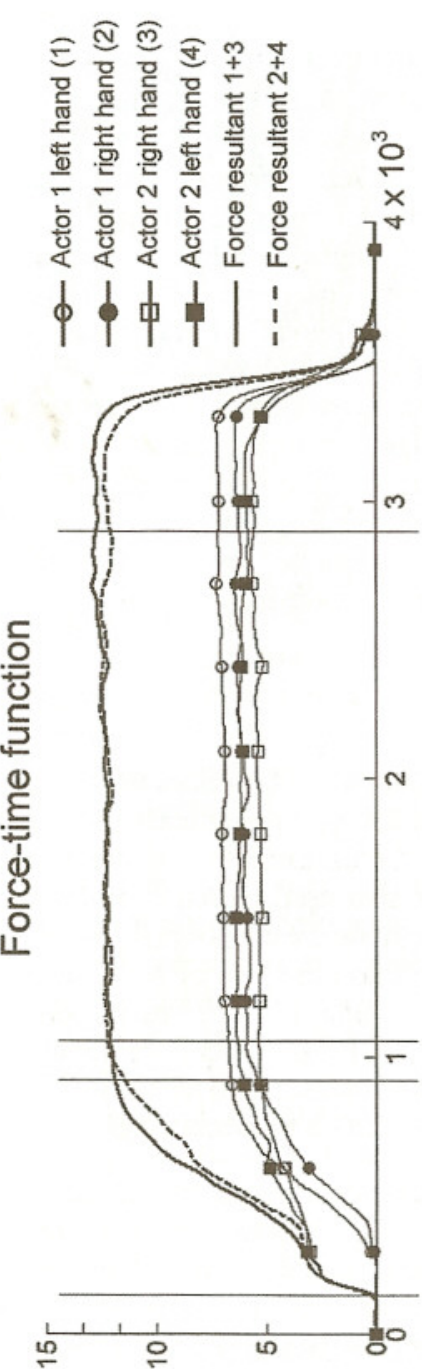

(N) әองดู

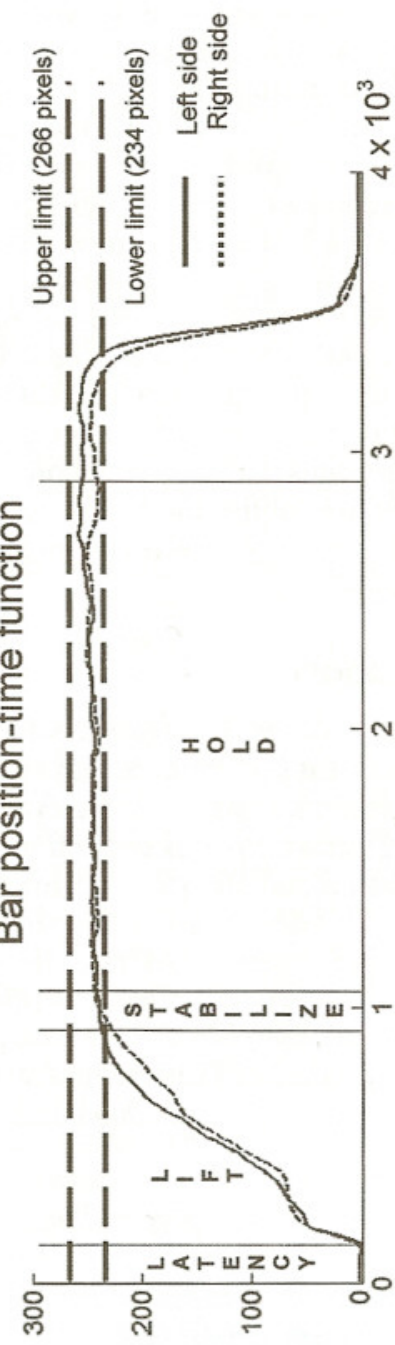

(x!̣) uo!̣!!sod teg gradually during the full hold phase until the performance criterion of the hold phase (i.e., maintaining the bar at the correct position for $2 \mathrm{~s}$ ) was reached.

The forces shown in the top panel of this figure resulted in positional changes of the bar-ends as shown in the bottom panel. The bar started moving after a latency of about $150 \mathrm{~ms}$ after the go-signal was given. Once both bar-ends were put into motion, the bar was lifted rapidly until both bar-ends approached the target area. Duration of the lifting phase was approximately $900 \mathrm{~ms}$. After stabilizing the bar into the target area $(\sim 100 \mathrm{~ms})$ the participants held the bar within the target area for the predefined 2-s period (holding phase).

\section{Success Rate}

Task performance was successful in $85 \%$ of the trials. For all dyads $(N=10)$ successful trials outnumbered unsuccessful ones (sign test, $N=10, p<.05$ ). On average, success rates for the 1-actor/2-hands, 2 -actors/2-hands, and 2-actors/4-hands conditions were $87.75 \%, 86.31 \%$, and $93.47 \%$, respectively.

For the following results we used the data set containing trials that were performed successfully.

\section{Single and Non-redundant Joint Action}

Targeted comparisons between the 1-actor/2-hands and 2-actors/2-hands conditions (Table 1) showed that reaction times for these two conditions were statistically indistinguishable [Duration; $t(9)=0.561$, ns] while synchronization was significantly stronger for individuals than for dyads performing with two hands [ $\triangle \mathrm{RT} ; \mathrm{t}$ $(9)=9.023, p<.01]$. Individuals, as opposed to dyads, were able to combine a fast lift [Duration; $\mathrm{t}(9)=5.115, p<.01$ ] with low variability in bar orientation [SDO; $\mathrm{t}(9)=6.710, p<.01]$ during the lifting phase. Table 1 shows that enhanced lifting performance by individuals is also reflected in cross-correlations and continuous relative phase analyses, i.e., relationships between the left and right bar-ends were strongly positive [Pearson; $\mathrm{t}(9)=11.434, p<.01]$, phase differences were smaller $[\mathrm{M} \phi ; \mathrm{t}(9)=2.963, p<.05]$ and relatively more stable $[S D \phi ; \mathrm{t}(9)=5.235, p<.01]$ in individuals than in dyads. While targeted comparisons showed that cycle time for positional change of the bar-ends for these two conditions were statistically indistinguishable [Cycle Time; $\mathrm{t}(9)=1.322, \mathrm{~ns}$ ], the basic frequency of corrective movements of the bar position during the lifting phase was significantly higher for individuals than for dyads performing with two hands [F0; $\mathrm{t}(9)=3.197, p<.05]$. Also during the stabilization phase, individuals performed better than dyads (Table 1). Individuals were able to stabilize the bar faster [Duration; $\mathrm{t}(9)=4.372, p<$ $.01]$ and showed smaller bar-orientation variability [SDO; $\mathrm{t}(9)=7.324, p<.01$ ] than dyads. Table 1 also shows that the correlations between the left and right barends were uncoupled in both individuals [Pearson; $\mathrm{r}(40)=+0.26, \mathrm{~ns}]$ and dyads [Pearson; $\mathrm{r}(40)=+0.08, \mathrm{~ns}]$ while cycle time between the targeted comparisons were statistically indistinguishable for these two conditions [Cycle Time; $t(9)=$ $1.240, n s]$. During the holding phase bar variability was slightly, but significantly, lower in individual performance than in performance by dyads $[S D O ; \mathrm{t}(9)=7.324$, $p<.01]$. Furthermore, relationships between the left and right bar-ends were more strongly positive in single than in joint action [Pearson; $\mathrm{t}(9)=11.195, p<.01$ ] Phase differences between bar-ends were smaller $[\mathrm{M} \phi ; \mathrm{t}(9)=8.075, p<.01]$ and 


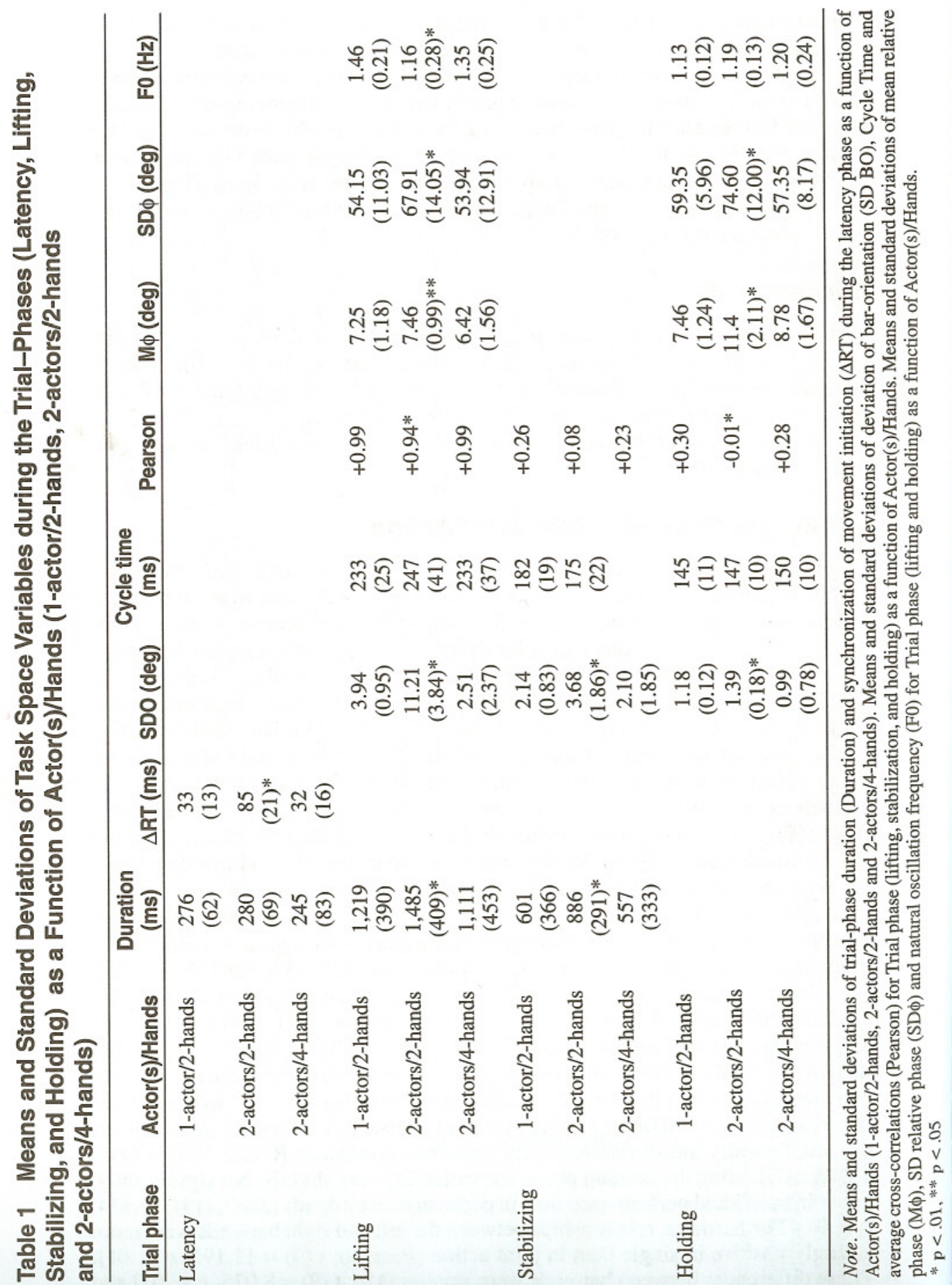

relatively more stable $[S D \phi ; \mathrm{t}(9)=5.195, p<.01]$ for individuals than for dyads while cycle time for positional change of the bar-ends [Cycle Time; $t(9)=1.571$, $\mathrm{ns}$ ] and the basic frequency of corrective movements of the bar position during the holding phase $[\mathrm{F} 0 ; \mathrm{t}(9)=1.235, \mathrm{~ns}]$ were statistically indistinguishable for these two conditions.

During lifting, the mean phase differences $(\mathrm{M} \phi)$ for positional changes between bar-ends in individual performance (Table 2) were comparable across the three grouped repetitions while the stability of these phase relationships increased from GR1 to GR2 [SD $;$; $(9)=3.574, p<.01]$ and were statistically indistinguishable between GR2 and GR3 [SD $\phi ; \mathrm{t}(9)=0.731$, ns]. In dyads, these phase differences decreased significantly from GR1 to GR2 $[\mathrm{M} \phi ; \mathrm{t}(9)=2.461, p<.05]$ while GR2 and GR3 were statistically indistinguishable $[\mathrm{M} \phi ; \mathrm{t}(9)=0.173$, ns]. The stability of these phase relationships increased significantly from GR2 to GR3 [SD; t (9) $=2.929, p<.05]$ while GR1 and GR2 were statistically indistinguishable [SD $\phi ; \mathrm{t}$ $(9)=1.851, \mathrm{~ns}]$ during lifting.

Table 2 Means $(\mathrm{M} \phi)$ and Standard Deviations (STD $\phi)$ of the Continuous Relative-Phase Signals of the Position-Change Function for Grouped Repetitions (GR1, GR2, and GR3) During Trial Phases (Lifting and Holding) as a Function of Actor(s)/Hands (1-actor/2-hands, 2-actors/2-hands and 2-actors/4-hands)

\begin{tabular}{|c|c|c|c|c|}
\hline $\begin{array}{l}\text { Trial } \\
\text { phase }\end{array}$ & Actor(s)/Hands & & M $\phi$ (deg) & SD $\phi$ (deg) \\
\hline \multirow[t]{9}{*}{ Lifting } & \multirow[t]{3}{*}{ 1-actor/2-hands } & GR1 & $9.45(3.03)$ & 60.23 (11.73) \\
\hline & & GR2 & $8.41(1.96)$ & $50.60(10.90)^{*}$ \\
\hline & & GR3 & $8.20(2.02)$ & $51.95(13.45)$ \\
\hline & \multirow[t]{3}{*}{ 2-actors/2-hands } & GR1 & $15.02(5.34)$ & 75.63 (12.97) \\
\hline & & GR2 & $10.15(5.02)^{* *}$ & 69.40 (15.96) \\
\hline & & GR3 & 9.93 (2.75) & $61.44(16.01)^{* * *}$ \\
\hline & \multirow[t]{3}{*}{ 2-actors/4-hands } & GR1 & $7.14(2.90)$ & 61.81 (18.95) \\
\hline & & GR2 & $6.81(1.49)$ & $51.69(13.55)^{* * *}$ \\
\hline & & GR3 & $6.06(2.02)$ & $51.48(12.10)$ \\
\hline \multirow[t]{9}{*}{ Holding } & \multirow[t]{3}{*}{ 1-actor/2-hands } & GR1 & 7.54 (2.65) & $63.26(5.77)$ \\
\hline & & GR2 & 7.49 (2.26) & 58.97 (9.94) \\
\hline & & GR3 & $7.82(2.08)$ & 57.05 (11.61) \\
\hline & \multirow[t]{3}{*}{ 2-actors/2-hands } & GR1 & $12.75(4.41)$ & 73.44 (14.47) \\
\hline & & GR2 & 11.49 (3.32) & $74.02(12.86)$ \\
\hline & & GR3 & $12.01(2.77)$ & 75.01 (11.45) \\
\hline & \multirow[t]{3}{*}{ 2-actors/4-hands } & GR1 & $9.92(1.64)$ & $62.19(5.46)$ \\
\hline & & GR2 & $8.13(2.77)$ & $52.43(10.98)^{* * *}$ \\
\hline & & GR3 & $8.89(2.14)$ & $53.74(12.96)$ \\
\hline
\end{tabular}


During the holding phase, phase differences for positional changes between bar-ends for individuals between GR1, GR2, and GR3 and the stability of these phase relationships GR1, GR2, and GR3 remained comparable across the grouped repetitions. Also, phase differences for dyads between GR1, GR2, and GR3 and the stability of the phase relationships GR1, GR2, and GR3 remained comparable across the grouped repetitions.

In sum, both individuals and dyads were equally fast in preparing and initiating the movement in the targeted conditions. However, individuals were faster than dyads in lifting and stabilizing the bar, exerting faster movement corrections during the lifting phase. Overall relationships between both bar-ends were stronger positive, with smaller phase differences and were performed relatively more stable by individuals than by dyads. Furthermore, phase relationships for positional changes between bar-ends during lifting showed systematic changes over trial repetitions.

\section{Redundant Joint Action}

Overall performance by dyads executing the task with four hands was assessed (see Table 1) with respect to movement initiation, synchronization of movement initiation, lifting time, and stabilization time, balancing of both bar-ends during the lifting, stabilization, and holding phases.

Correlations between bar-ends were, on average, strongly positive [Pearson; $\mathrm{r}$ $(70)=+0.99, p<.01]$ during the lift, uncoupled during stabilization $[\mathrm{r}(40)=+0.23$, $\mathrm{ns}]$ and weakly positive during holding [Pearson; $\mathrm{r}(70)=+0.28, p<.01]$. Phase differences of positional changes between bar-ends were relatively small during lifting and relatively larger during holding. These relationships were relatively more stable during the lifting phase than during the holding phase. Cycle time of the position-change function of the bar was higher for lifting than for holding the bar whereas the basic frequency of the corrective movements of the bar were in the same order for the lifting and holding phase.

In the lifting phase (see Table 2), the $\mathrm{M} \phi$ for positional changes between barends was comparable across the grouped repetitions while the stability of these phase relationships increased from GR1 to GR2 [SD $\phi ; \mathrm{t}(9)=3.018, p<.05]$ and were statistically indistinguishable between GR2 and GR3 [SD ; t $(9)=0.057, \mathrm{~ns}$ ]

During the holding phase, phase differences for positional changes between bar-ends remained comparable across the grouped repetitions. The stability of these phase relationships showed an increase from GR1 to GR2 [SD ; t $(9)=2.512, p$ $<.05$ ], while the $S D \phi$ between GR2 and GR3 were statistically indistinguishable $[S D \phi ; \mathrm{t}(9)=0.297, \mathrm{~ns}]$.

These results show that dyads in redundant joint action combined a relative fast task execution with a low variability in bar orientation. They displayed a systematic increase in stable phase relationships across grouped repetitions in the lifting and holding phases. Overall, relationships between bar-ends were strongly positive, displayed smaller phase differences and were relatively more stable during lifting than holding the bar. Furthermore, low-frequency action monitoring feedback loops were on the order of $1 \mathrm{~Hz}$ during the lift and hold phases.

Figure 3 shows force relationships of a single trial that reflect the typical performance of dyads performing with four hands. During the holding phase, forces of both hands of Actor 1 decreased simultaneously while, at the same time, forces of

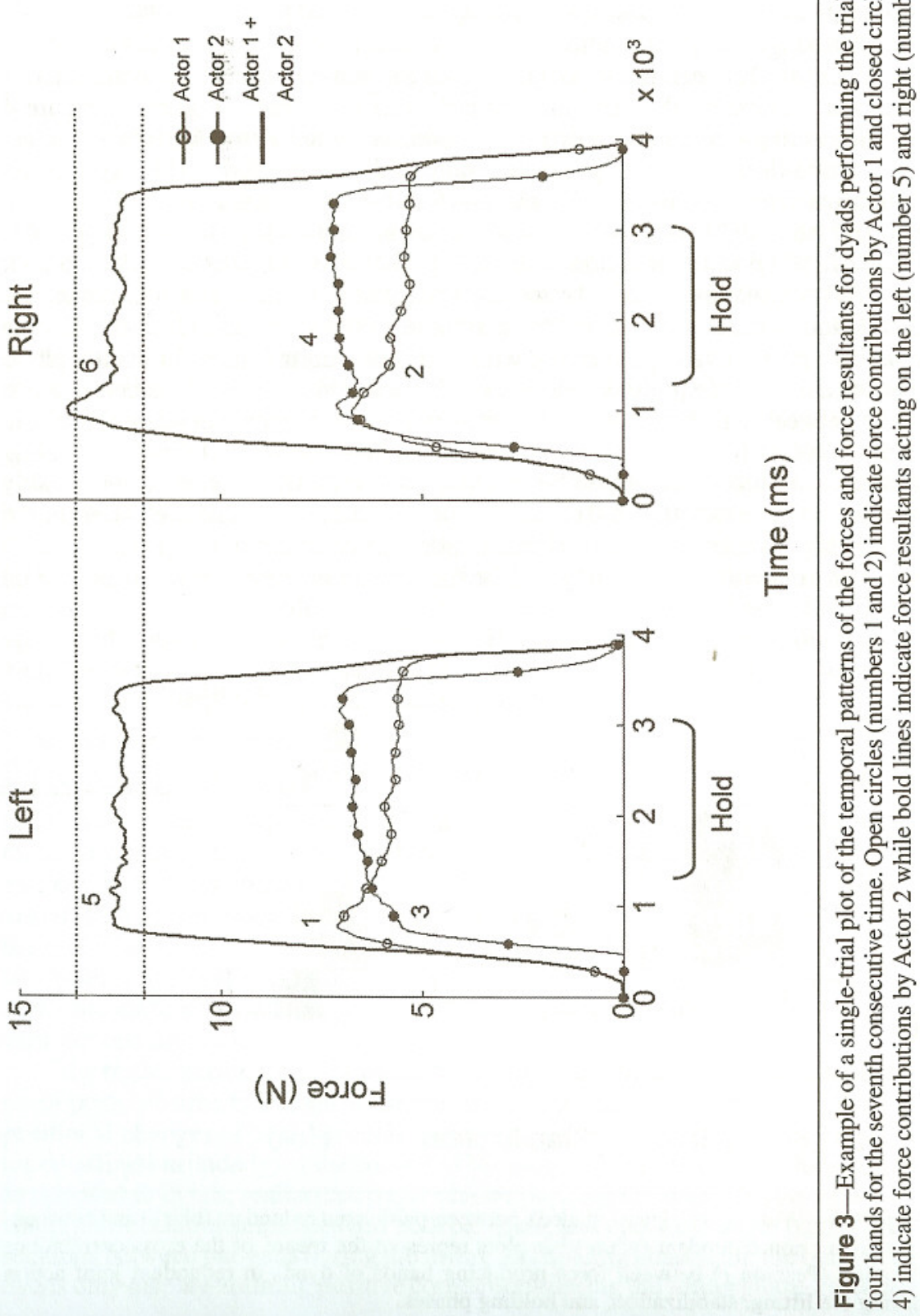


both hands of Actor 2 increased synchronously resulting in reciprocally gradually changing supernumerary force contributions. The figure displays strong negative correlations between supernumerary forces between actors: $[\mathrm{r}(70)=-0.90, p<$ $.01]$ in the left panel and $\operatorname{rr}(70)=-0.80, p<.01]$ in the right panel. Furthermore, correlations between both hands of Actor $1[\mathrm{r}(70)=+0.92, p<.01]$ and Actor $2[\mathrm{r}$ $(70)=+0.86, p<.01]$ were strongly positive while correlations between the force resultants were weakly positive $[\mathrm{r}(70)=+0.39, p<.01]$.

This single-trial observation is substantiated by the overall results of withinparticipant and between-participant redundant and non-redundant force contributions of dyads performing the experimental task with four hands (Figure 4). Figure 4 shows the cross-correlations between the contributing forces for dyads in redundant joint action during the lifting, stabilization, and holding phases. During the lift, within-participant correlations in the 2 -actors/4-hands condition $[\mathrm{r}(70)=+0.98$, $p<.01]$ were higher than between-participant redundant $[\mathrm{r}(70)=+0.93, p<.01$; $\mathrm{t}(9)=7.225, p<.01]$ and non-redundant $[\mathrm{r}(70)=+0.92, p<.01 ; \mathrm{t}(9)=6.553$, $p<.01]$ correlations, while between-participant redundant and non-redundant correlations were statistically indistinguishable $[\mathrm{t}(9)=1.653, \mathrm{~ns}]$. On average, force relationships for dyads performing with four hands during the stabilization phase were weakly positive $[\mathrm{r}(40)=+0.33, p<.05]$ for the within-participant correlations, while between-participant redundant $[\mathrm{r}(40)=+0.08$, ns] and between-participant non-redundant $[\mathrm{r}(40)=-0.18, \mathrm{~ns}]$ correlations were absent. During the holding phase, correlations for the within-participant forces were, on average, weakly positive $[\mathrm{r}(70)=+0.46, p<.01]$ and significantly different than correlations for the weakly negative between-participant redundant force contributions $[\mathrm{r}(70)=-0.27$, $p<.05 ; \mathrm{t}(9)=4.447, p<.01]$ and for the weakly negative between-participant

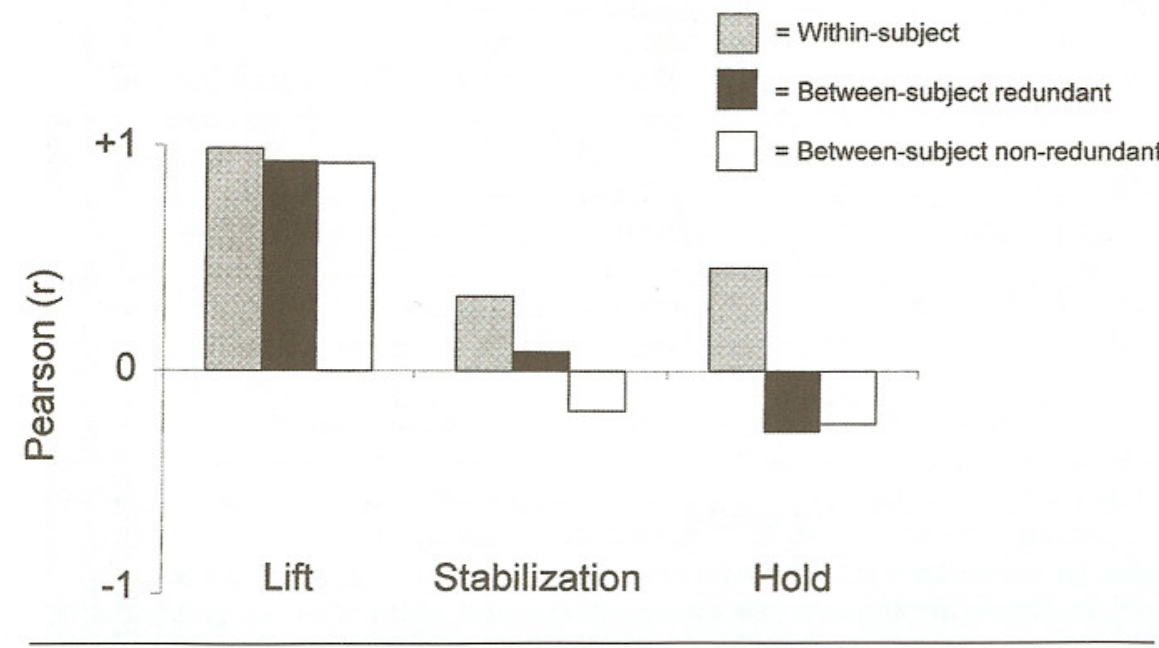

Figure 4-Within-participant (shaded), between-participant redundant (black) and betweenparticipant non-redundant (white) bar plots represent the means of the cross-correlations function (Pearson $r$ ) between force-producing hands of dyads in redundant joint action during the lifting, stabilization, and holding phases. non-redundant forces $[\mathrm{r}(70)=-0.24, p<.05 ; \mathrm{t}(9)=3.979, p<.01]$. Correlations for between-participant redundant forces and non-redundant forces were statistically indistinguishable [t $(9)=1.116$, ns].

In sum, within-participant relationships between force contributions of dyads performing with four hands ranged from highly positive to weakly positive while between-participant relationships ranged from highly positive during the lifting phase to weakly negative during the holding phase.

\section{Discussion}

In this study, we investigated redundancy control when two people performed an isometric force-production, virtual bar-lifting task. Participants executed the isometric lifting task alone bimanually and together either with two or with all four hands and could not see each other and were not allowed to verbally communicate. Not only did individuals and dyads have to transport the bar into a target area and hold it there for a 2-s period, but unbalanced force generation resulted in a tilt (rotation) of the bar in the frontal plane. Our aim was to assess the features of the individual and group contributions to the action and to capture the temporal characteristics of their coordinated attempt to adjust both the height and rotation of the bar in their task performance. In performing the isometric lifting task with four hands, the system was overspecified and task-specific structural units (synergies) between the force-producing hands were presumed to be created. One may hypothesize that at the level of movement planning these structural units simplify the control problem. The present findings provide, in our view, an indication that lacking a shared neural substrate and diminished sensory information does not necessarily present individuals with an unbridgeable gap to work together. Under such conditions dyads can create task-specific structural units, even though group members will execute tasks that they perform together slower than individuals do and may perform poorly on synchronizing their actions.

\section{Single and Non-redundant Joint Action}

Individuals were, as expected, better in synchronizing both bar-ends after movement initiation than dyads, and outperformed dyads in lifting and stabilizing the bar, exerting faster movement corrections during the lifting phase; individuals also displayed more skill in controlling the tilt of the bar throughout all phases of the trial (Table 1). These observations are in line with results in previous studies (Burstedt et al., 1997; Schmidt et al., 1998; Knoblich et al., 2003) that have shown performance to be impeded in joint action if the task at hand requires dyads to time their actions together.

The higher performance by individuals is reflected by cross-correlations analysis of position-time functions of the bar and continuous relative phase analysis of positional changes of both bar-ends that were applied to uncover the dynamics of the coordination underlying the control of bar movements (see Table 1). Individuals, as opposed to dyads, realized stronger phase coupling, maintained relatively more stable phase relationships between both bar-ends, and upheld stronger positive correlations between bar-ends during the lifting and holding phases. On the other hand, dyads only displayed strong positive correlations during the lifting phase. However, 
the strong positive correlations during the lift hardly provide strong evidence of synchronized joint action. For example, sprinters participating in the $100 \mathrm{~m}$ finals of a race event will all be out of the starting blocks in roughly the same time after the starting signal but obviously have no interest in intentionally coordinating their actions among each other during the race. We therefore take these results to reflect performance that is dictated by the synchronized "go" signal while aiming for a common goal (see also Figure 4).

A possible explanation for enhanced performance in individuals can be found by presupposing that they received both fast haptic feedback loops from tactile afferents of the fingertips and relatively slow online visual feedback of the bar displacements on the computer screen while dyads could only rely on relatively slow online visual feedback of their partners' actions to perceive the consequences of their actions. However, cycle time, as a measure of repetitive tuning of positional changes of the bar-ends, was in the same order within each trial phase for both individuals and dyads (see Table 1) indicating that individuals also mainly relied on visual-motor feedback loops to monitor their actions. Because the two hands of the individual are anatomically linked they were able to realize a proactive coordination regime and execute corrective movements more often during the lifting phase than dyads.

Even though dyads do not share a common neural substrate, they were as successful as individuals in performing the experimental task. Moreover, dyads also displayed adaptive behavior over trial repetitions during the lifting phase be it that dyads were slower in adapting to the requirements of the task dynamics than individuals (see Table 2). While individuals quickly learned to produce mor stable interactions between both bar-ends over trial repetitions, dyads responded first by reducing phase differences between contributing hands and at a later stage increased the stability of these interactions.

Surprisingly, both individuals and dyads in the two-handed conditions were equally fast in preparing and initiating the movement. We use the word "surprisingly" because performance by dyads in the two-handed condition could, technically speaking, be viewed as a one-handed performance by two separate actors and unilateral movements have been shown to take shorter to initiate than simultaneous bilateral single-actions carried out by two hands (Kelso et al., 1979; Ohtsuki, 1994). Swinnen and Wenderoth (2004) have argued that within the information-processing perspective, dual-task performance by one brain is faced with structural interference. This concept of neural crosstalk presupposes that information leakage may occur at different levels of the central nervous system (cortical to spinal), both during bimanual motor programming and execution. Consequently, during moto programming and initializing of the movement, dyads in the two-handed condition must have taken each others' preparation for the task into account. We find support for our observation in a number of studies concerning discrete tasks, showing that an efficient means to predict others' actions that is not necessarily based on action observation but knowing what another's task is (Sebanz \& Frith, 2004; Sebanz, Knoblich \& Prinz, 2005). By forming shared task representations, it is possible to predict actions based on certain events in the environment, independent of action observation (Sebanz, Bekkering \& Knoblich, 2006).

\section{Redundant Joint Action}

An expected key finding in this study is that correlations between supernumerary force contributions by dyads performing the task with four hands, during the holding phase, were found to be negative (Figure 4). This means that interacting partners not only responded to observed changes but also incorporated the timing of the actions of the partner in their own action planning. This feature of joint action is remarkable if we take into consideration that the contributions to the lifting task by one partner were not directly observable for the other, i.e., dyads acted on relatively slow online visual feedback of the composite adjustments that they jointly brought about.

Of course, dyads would not necessarily have to remain ignorant of their partner's contribution to the task. For example, by first giving an arbitrary battery of force outputs for system identification purposes and then by generating error measures from the internal predictions of their own movements (Wolpert \& Ghahramani, 2000) and the observed joint-movements on screen, the error measures would allow them to assess their partner's contribution to the task. It is obvious that the type of coordination that evolves from this course of action is reactive in nature (i.e., successive actions are produced on the basis of inferring the composite feedback) and therefore may not seem to provide a means of modeling synchronized action coordination in redundant joint action.

Both intentional synchronization of movements (Schmidt et al., 1994) and unintentional synchronization of movements (Schmidt et al., 1990; Schmidt \& O'Brien, 1997) have been observed for the swinging of hand-held pendulums by pairs of participants when they can see each other's movements and modeled by the Haken, Kelso, and Bunz (1985) model and its modifications (Kelso \& Jeka, 1992; Fuchs \& Kelso, 1994). However, it is not clear how the Haken et al. (1985) model is able to deal with the absence of direct visual feedback of the partners' contributions to the task (see also Rosenbaum et al., 2006).

Negative correlations during the holding phase could also indicate that the task at hand is to be viewed as a tracking task. This is because now that the bar is in the proper place with the proper orientation, each person is visually "tracking" the other; in other words, we have compensatory tracking and of course there will be a negative correlation between the error and the correction to it, which is the definition of negative feedback.

Because the output to the lifting task was composed of both actors' actions, dyads had no direct feedback of their partner's contributions to the task. This means that dyads in redundant conditions can only compensate for the error they mutually bring about, thereby ruling out the possibility that each person "tracks" the other and thereby the presumption that the task during the holding phase can be viewed as a compensatory tracking task.

On the other hand, research has also shown that actors can learn to make accurate force-related predictions (Dizio \& Lackner, 1995; Shadmehr \& Mussa-Ivaldi, $1994)$ as well as adaptive anticipatory changes to altered visual feedback, as in prism adaptation (Redding \& Wallace, 1997) by forming an internal model of the joint-dynamics of the action of which they are a part (cf. Atkeson, 1989; Jordan, 1994). We assume that adaptive behavior would therefore enable dyads to generate 
forces that anticipate rather than merely react to the actions of their counterpart.

Our results show (Table 2) that dyads performing redundant joint action quickly increased stable phase relationships between both bar-ends over trial repetitions in both the lifting and holding phases. This implies that dyads were quite capable of displaying adaptive behavior by the contributing forces in controlling both dimensions of the bar in the course of trial repetitions.

The present results did not reveal a joint-action coordination strategy of perfect degree-of-freedom allocation across the participants, i.e., one actor taking care of the height of the bar and the other controlling its rotation. Dyads performing the task with four hands could have developed a cooperative strategy such that one member contributed more to the height and the other to constraining the rotation of the bar. However, Figure 4 shows that those dyads created three synergies that operated in synchrony during the holding phase. Each of the actors created a separate synergy by coupling positional changes of bar-ends to control the bar orientation, and at the same time, these synergies were inversely coupled to jointly control the bar height. These findings underscore our conclusion that, in the redundant joint action, both actors show a strong tendency to take their share in controlling both task dimensions in order to perform the task at hand successfully.

Typically, the generated forces increased or decreased very gradually throughout the holding phase, as can be observed in the single-trial plots shown in Figure 3. Analyses of autocorrelation functions revealed that the bar-orientation time functions displayed an average periodicity of $1.17 \mathrm{~Hz}$. We take that these regularly recurring changes in bar orientation are indicative of low-frequency feedback loops that subserve action-monitoring functions while holding the bar in place. Studies in which the intermittency of visual feedback was manipulated while people tried to maintain a steady force level have shown that action-monitoring functions in individuals also seem to be governed by low-frequency feedback loops (Slifkin, Vaillancourt \& Newell, 2000; Sosnoff \& Newell, 2005). We hypothesize that the gradual changes in bar-orientation in the present study are due to positional drift as an effect of diminished visual feedback. Research into hand-position matching have shown that without vision, the accuracy with which finger locations are reported declines over repeated matches such that perception of limb position appears to drift (Paillard \& Brouchon, 1968; Wann \& Ibrahim, 1993; Wolpert, Goodbody \& Husain, 1998). Due to the gradual nature of the changes in bar-orientation during the holding phase in this experiment, actors are provided with ample time to effectively plan their action over time and counterbalance for the positional drift by low-frequency modulated forces to keep their performance tuned to task demands.

In general, our results show that the coupling of effectors does not depend on whether their neural control centers are anatomically linked and confirms earlier suggestions that such coupling may be informationally and not just anatomically based. Furthermore, the results indicate the presence of homogeneously attuned forces, synchronized by the "go" signal to make a common cause in lifting, that the forces were reactive in nature during the stabilization phase to serve as an upbeat for holding the bar in place where inverse force relationships between supernumerary force contributions indicate that dyads were capable of resolving redundancy by administering force-sharing synergies that, in our view, certifies the signature of synchronized joint-action in our experimental task.

\section{Acknowledgments}

The present study was supported by the EU Project "Joint Action Science and Technology" (IST-FP6-003747). We thank Majken Hulstijn for her generous contribution in acquiring data for this experiment, Hubert Voogd and Raymond Cuijpers for technical assistance, and the partners on the JAST project for helpful discussions. We also thank those who kindly volunteered to participate in the study.

\section{References}

Atkeson, C.G. (1989). Learning arm kinematic and dynamics. Annual Review of Neuroscience, 12, 157-183.

Amazeen, P.G., Schmidt, R.C., \& Turvey, M.T. (1995). Frequency detuning of the phase entrainment dynamics of visually coupled rhythmic movements. Biological Cybernetics, 72, 511-518.

Batschelet, E. (1981). Circular statistics in biology. London: Academic Press.

Bernstein, N. (1967). The coordination and regulation of movements. London: Pergamon. Burstedt, M.K.O., Edin, B.B., \& Johansson, R.S. (1997). Coordination of fingertip forces during human manipulation can emerge from independent neural networks controlling each engaged digit. Experimental Brain Research, 117, 67-79.

Carlton, L.G. (1981). Processing visual feedback information for movement control. Journal of Experimental Psychology: Human Perception and Performance, 7, 1019-1030.

Conditt, M.A., Gandolfo, F., \& Mussa-Ivaldi, F.A. (1997). The motor system does not learn the dynamics of the arm by rote memorization of past experience. Journal of Neurophysiology, 78, 554-560.

Cole, K.J., \& Abbs, J.H. (1986). Coordination of three-joint digit movements for rapid finger-thumb grasp. Journal of Neurophysiology, 55, 1407-1423.

Danion, F., Schöner, G., Latash, M.L., Li, S., Scholz, J.P., \& Zatsiorsky, V.M. (2003). A force mode hypothesis for finger interaction during multi-finger force production tasks. Biological Cybernetics, 88, 91-98.

D'Avella, A., Saltiel, P., \& Bizzi, E. (2003). Combinations of muscle synergies in the construction of a natural motor behavior. Nature Neuroscience, 6, 300-308.

Dizio, P., \& Lackner, J.R. (1995). Motor adaptation to coriolis force perturbations of reaching movements: Endpoint but not trajectory adaptation transfers to the nonexposed arm. Journal of Neurophysiology, 74, 1787-1792.

Flash, T., \& Hogan, N. (1985). The coordination of arm movements: An experimentally confirmed mathematical model. Journal of Neuroscience, 5, 1688-1703.

Ferbert, A., Priori, A., Rothwell, J.C., Day, B.L., Colebatch, J.G., \& Marsden, C.D. (1992). Interhemispheric inhibition of the human motor cortex. Journal of Physiology, 453, 525-546.

Fuchs, A., \& Kelso, J.A. (1994). A theoretical note on models of interlimb coordination. Journal of Experimental Psychology: Human Perception and Performance, 20, 1088-1097.

Gazzaniga, M.S., \& Sperry, R.W. (1966). Simultaneous double discrimination response following brain bisection. Psychonomic Science, 4, 261-262.

Haken, H., Kelso, J.A., \& Bunz, H. (1985). A theoretical model of phase transitions in human hand movements. Biological Cybernetics, 51, 347-356.

Hakkinen, K., Kraemer, W.J., \& Newton, R.U. (1997). Muscle activation and force production during bilateral and unilateral concentric and isometric contractions of the knee extensors in men and women at different ages. Electromyography and Clinical Neurophysiology, 37, 131-142. 
Harris, C.M. (1998). On the optimal control of behaviour: a stochastic perspective. Journal of Neuroscience Methods, 83, 73-88.

Harris, C.M., \& Wolpert, D.M. (1998). Signal-dependent noise determines motor planning. Nature, 394, 780-784.

Ivanenko, Y.P., Grasso, R., Zago, M., Molinari, M., Scivoletto, G., Castellano, V., Macellari, V., \& Lacquaniti, F. (2003). Temporal components of the motor patterns expressed by the human spinal cord reflect foot kinematics. Journal of Neurophysiology, 90, $3555-3565$.

Johansson, R.S., \& Birznieks, I. (2004). First spikes in ensembles of human tactile afferents code complex spatial fingertip events. Nature Neuroscience, 7, 170-177.

Jordan, M.I. (1994). Computational motor control. In M.S. Gazzaniga (Ed.). The cognitive neurosciences (pp. 597-609). Cambridge, MA: MIT Press.

Kelso, J.A., \& Jeka, J.J. (1992). Symmetry breaking dynamics of human multilimb coordination. Journal of Experimental Psychology: Human Perception and Performance, 18, 645-668.

Kelso, J.A., Southard, D.L., \& Goodman, D. (1979). On the coordination of two-handed movements. Journal of Experimental Psychology: Human Perception and Performance, 5, 229-238

Knoblich, G., \& Jordan, J.S. (2003). Action coordination in groups and individuals: Learning anticipatory control. Journal of Experimental Psychology: Learning, Memory, \& Cognition, 29, 1006-1016.

Koh, T.J., Grabiner, M.D., \& Clough, C.A. (1993). Bilateral deficit is larger for step than for ramp isometric contractions. Journal of Applied Physiology, 74, 1200-1205.

Li, Z.M., Latash, M.L., \& Zatsiorsky, V.M. (1998). Force sharing among fingers as a model of the redundancy problem. Experimental Brain Research, 119, 276-286.

Meulenbroek, R.G.J., Thomassen, A.J.W.M., Van Lieshout, H.P.M., \& Swinnen, S.P. (1998). The stability of pen-joint and interjoint coordination in loop writing. Acta.Psychologica, 100, $55-70$.

Morasso, P. (1981). Spatial control of arm movements. Experimental Brain Research, 42, 223-227.

Nakano, E., Imamizu, H., Osu, R., Uno, Y., Gomi, H., Yoshioka, T., Kawato, M. (1999). Quantitative examinations of internal representations for arm trajectory planning: minimum commanded torque change model. Journal of Neurophysiology, 81, 2140-2155.

Oda, S. \& Moritani, T. (1994). Maximal isometric force and neural activity during bilateral and unilateral elbow flexion in humans. European Journal of Applied Physiology, 69, 240-243.

Oda, S. \& Moritani, T. (1995). Cross-correlation of bilateral differences in fatigue during sustained maximal voluntary contraction. European Journal of Applied Physiology, 70, 305-310.

Ohtsuki, T. (1994). Changes in strength, speed, and reaction time induced by simultaneous bilateral muscular activity. In S.P. Swinnen, H. Heuer, J. Massion, \& P. Casaer (Eds.), Interlimb coordination: neural, dynamical and cognitive constraints (pp. 259-274). San Diego, CA: Academic Press.

Oldfield, R.C. (1971). The assessment and analysis of handedness: the Edinburgh Inventory. Neuropsychologia, 3, 97-113.

Paillard, J., \& Brouchon, M. (1968). Active and passive movements in the calibration of position sense. In S.J. Freedman (Ed.). The neuropsychology of spatially oriented behavior (pp. 37-55). Homewood, IL: The Dorsey Press.

Redding, G.M., \& Wallace, B. (1997). Adaptive spatial alignment. Mahwah, NJ: Lawrence Erlbaum Associates.

Rosenbaum, D.A., Loukopoulos, L., Meulenbroek, R.G.J., Vaughan, J., \& Engelbrecht, S.E., (1995). Planning reaches by evaluating stored postures. Psychological Review, 102, 28-67.
Rosenbaum, D.A., Meulenbroek, R.G.J., Jansen, C., \& Vaughan, J. (2001). Posture-based motion planning: Applications to grasping. Psychological Review, 108, 709-734.

Rosenbaum, D.A., Dawson, A.M., \& Challis, J.H. (2006). Haptic tracking permits bimanual independence. Journal of Experimental Psychology: Human Perception and Performance, 32, 1266-1275.

Santello, M., Flanders, M., \& Soechting, J.F. (1998). Postural hand synergies for tool use. Journal of Neuroscience, 18, 10105-10115.

Santello, M., \& Soechting, J.F. (2000). Force synergies for multifingered grasping. Experimental Brain Research, 133, 457-467.

Saunders, J.A., \& Knill, D.C. (2003). Humans use continuous visual feedback from the hand to control fast reaching movements. Experimental Brain Research, 152, 341-352.

Shadmehr, R., \& Mussa-Ivaldi, F.A. (1994). Adaptive representation of dynamics during learning of a motor task. The Journal of Neuroscience, 14, 3208-3224.

Schieber, M.H., \& Santello, M. (2004). Hand function: peripheral and central constraints on performance. Journal of Applied Physiology, 96, 2293-2300.

Schmidt, R.C., Carello, C., \& Turvey, M.T. (1990). Phase transitions and critical fluctuations in the visual coordination of rhythmic movements between people. Journal of Experimental Psychology: Human Perception \& Performance, 16, 227-247.

Schmidt, R.C., \& Turvey, M.T. (1994). Phase-entrainment dynamics of visually coupled rhythmic movements. Biological Cybernetics, 70, 369-376.

Schmidt, R.C., \& O'Brien, B. (1997). Evaluating the dynamics of unintended interpersonal coordination. Ecological Psychology, 9, 189-206.

Schmidt, R.C., Bienvenu, M., Fitzpatrick, P.A., \& Amazeen, P.G. (1998). A comparison of intraand interpersonal interlimb coordination: coordination breakdowns and coupling strength. Journal of Experimental Psychology: Human Perception \& Performance, 24, 884-900.

Sebanz, N., \& Frith, C. (2004). Beyond simulation? Neural mechanisms for predicting the actions of others. Nature Neuroscience, 7, 5-6.

Sebanz, N., Knoblich, G., \& Prinz, W. (2005). How two share a task: corepresenting stimulus-response mappings. Journal of Experimental Psychology: Human Perception \& Performance, 31, 1234-1246.

Sebanz, N., Bekkering, H., \& Knoblich, G. (2006). Joint action: bodies and minds moving together. Trends in Cognitive Science, 10, 70-76.

Slifkin, A.B., Vaillancourt, D.E., \& Newell, K.M. (2000). Intermittency in the control of continuous force production. Journal of Neurophysiology, 84, 1708-1718.

Sosnoff, J.J., \& Newell, K.M. (2005). Intermittent visual information and the multiple timescales of visual motor control of continuous isometric force production. Perception \& Psychophysics, 67, 335-344.

Swinnen, S.P., \& Wenderoth, N. (2004). Two hands, one brain: cognitive neuroscience of bimanual skill. Trends in Cognitive Sciences, 8, 18-25.

Uno, Y., Kawato, M., \& Suzuki, R. (1989). Formation and control of optimal trajectory in human multijoint arm movement. Minimum torque-change model. Biological Cybernetics, 61, 89-101.

Viviani, P., \& Flash, T. (1995). Minimum-jerk, two-thirds power law, and isochrony: converg ing approaches to movement planning. Journal of Experimental Psychology: Human Perception and Performance, 21, 32-53.

Wann, J.P., \& Ibrahim, S.F. (1993). Does limb proprioception drift? Experimental Brain Research, 91, 162-166.

Wolpert, D.M., Goodbody, S.J., \& Husain, M. (1998). Maintaining internal representations: the role of the human superior parietal lobe. Nature Neuroscience, 1, 529-533.

Wolpert, D.M., \& Ghahramani, Z. (2000). Computational principles of movement neuroscience. Nature Neuroscience, 3, 1212-1217.

Wyke, M. (1971). The effects of brain lesions on the learning performance of a bimanual co-ordination task. Cortex, 7, 59-72. 


\section{Appendix:}

\section{Criterion (Search Algorithm) for Latency Phase}

Starting at $\mathrm{t}=0$

Find for $\mathrm{n}=20$ successive samples $(13.33 \mathrm{~ms})$ of sampled force production (at $1,500 \mathrm{~Hz}$ ) of which:

1. Force production $<10 \%$ maximum force production

2. Time $<$ end of experiment

$\mathrm{Xm}=$ Mean force production of samples $\mathrm{i}, \mathrm{i}+1 . . \mathrm{i}+\mathrm{n}-1$

$\mathrm{Xsd}=S D$ force production of samples $\mathrm{i}, \mathrm{i}+1 . . \mathrm{i}+\mathrm{n}-1$

Latency $=$ Time delay between the visual go-signal (for $t=0)$ and averaged time indices of first samples at which force production $>\mathrm{Xm}+3 * \mathrm{Xsd}$ 